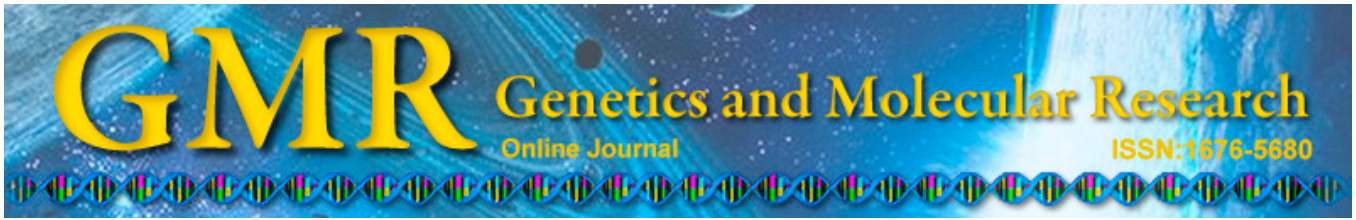

\title{
Telomere-associated factor expression in replicative senescence of human embryonic lung fibroblasts
}

\author{
H. Du ${ }^{1.2 *}$, L. Yang ${ }^{1 *}$, X.-Y. Xu ${ }^{1,2}$, L. Hai ${ }^{1,2}$, Y.-Q. Han ${ }^{1,2}$ and Y.-X. Shi ${ }^{1}$ \\ ${ }^{1}$ Affiliated Hospital of Inner Mongolia Medical University, Hohhot, China \\ ${ }^{2}$ Inner Mongolia Medical University, Hohhot, China \\ *These authors contributed equally to this study. \\ Corresponding authors: Y.-X. Shi / Y.-Q. Han \\ E-mail: shiyingxu@126.com / gyh1016@sina.com
}

Genet. Mol. Res. 14 (3): 9269-9276 (2015)

Received November 5, 2014

Accepted April 24, 2015

Published August 10, 2015

DOI http://dx.doi.org/10.4238/2015.August.10.7

\begin{abstract}
The objective of this study was to find the key regulatory molecules in the cell senescence process through observing the expression of telomere-associated factor during the normal cell replicative senescence process. Based on the established cell replicative senescence model, reverse transcription-polymerase chain reaction and western blot analyses were used to detect telomere-associated factor expression at the mRNA and protein levels, including that of human telomere binding protein 1 , tankyrase 1 , telomerase RNA, telomere protection protein 1 (POT1), and p53 during the process of human embryonic lung fibroblast replicative senescence. The results showed that transcription of human telomere binding protein 1 did not change with cell senescence, whereas the protein expression of human telomere binding protein 1 increased gradually and then decreased rapidly; there was no change in the mRNA and protein expression of POT1; with the replicative senescence of human embryonic lung fibroblasts, expression of POT1 decreased gradually; TRF1 showed an increasing trend with cell senescence; and p53 protein expression did not change. Together,
\end{abstract}


the results from this study suggest that human telomere binding protein 1, POT1, and TRF1 played important roles in cell senescence.

Key words: Replicative senescence; Telomerase RNA;

Telomere protection protein 1; Telomere related binding factor 1

\section{INTRODUCTION}

Cell senescence is both a major biological problem as well as a major social problem. Cell senescence is a phenomenon of functional decline under normal environmental conditions, gradually moving toward death. Studies on cell senescence can reveal the underlying mechanisms of senescence, which is important for understanding the phenomenon and potentially mediating its delay. Many studies have found that cell replicative senescence is closely related to telomere length: the shortening of telomeres is the cause of cell replicative senescence, and telomere length is in turn regulated by telomere-related factors. Therefore, it can be proposed that the relationships between cell telomere-associated factors and replicative senescence can reveal the mechanism of cell senescence.

Telomere related binding factor 1 (TRF1) and telomere protection protein 1 (POT1) are telomere-binding proteins; human telomere binding protein 1 and POT1 contact is established by the interactions of TRF1 interacting nucleoprotein (TIN), POT1 and POT2 organizing protein (PTOP), and POT1-interacting protein (PIP1) (Abreu et al., 2010; Takai et al., 2010, 2011; Xie et al., 2011). Lundblad (2003) considered that the binding of POT1 and ssDNA was mediated by telomerase-binding protein 1: in the cell, POT1 first combines with the human telomeric binding protein complex, followed by the perception of telomere length changes by human telomere binding protein 1, which guides the binding of POT1 and ssDNA. Many experiments have confirmed the contact of POT 1 with human telomere-binding protein 1 complexes (Blasco, 2003; Giannone et al., 2010; Martínez and Blasco, 2010; Choi et al., 2011; Miller et al., 2012).

p53 is a tumor suppressor gene and is one of the important regulatory genes for cell cycle and apoptosis. p53 is also an important regulatory factor in cell senescence and can perceive senescence-related signals such as telomere shortening, DNA damage, and over-expression of suppressor genes. Studies have found that p53 and retinoblastoma $(\mathrm{Rb})$ together maintain the inhibition state of cell growth. Decreased p53 activity can delay cell replicative senescence but cannot prevent it (Feng et al., 2011; Itahana et al., 2011). In the Ataxia telangiectasia mutated (ATM)/p53/cyclin dependent kinase inhibitor 1 (p21WAF-1) pathway, p53 can inhibit cell senescence by inducing p21WAF-1 expression, which was shown to have a positive correlation with p53 activity. Therefore, a study on the expression changes of TRF1, tankyrase 1, telomerase RNA, POT1, and p53 in normal cell replicative senescence can provide a theoretical basis for further studies on normal mechanisms of aging.

\section{MATERIAL AND METHODS}

\section{Material}

Human embryonic lung fibroblasts were provided by the Molecular Biology Laboratory, Institute of Radiation Medicine, Beijing Military Medical Academy. Cells were 
incubated with Dulbecco's modified Eagle's medium (DMEM) (Life Technologies, USA) containing $10 \%$ fetal bovine serum in an incubator at $37^{\circ} \mathrm{C}$ in an atmosphere containing $5 \% \mathrm{CO}_{2}$.

\section{Methods}

PCR primers were designed according to the gene sequences of TRF1, tankyrase 1, telomerase RNA, POT1-2, and $\beta$-actin as follows: TRF1 (F: 5'-GAA TAT TTG GTG ATC CAA ATT CTC ATA TGT-3'; R: 5'-AGT TAC CGC AGA CTG TTT GTC ACT AAC ACT3'); tankyrase 1 (F: 5'-CCG GAG GGG GAT GGC AGT CGG GAT CCG CCC-3'; R: 5'-CCG GCC GGC CAT GTC CTT TGC ATT TAC GTT-3'); telomerase RNA (F: 5'-CGA ACG GGC CAG CAG CTG ACA TT-3'; R: 5'-GGG TGG TGG CCA TTT TTT GT-3'); POT1-2 (F: 5'TTC AGA TGT TAT CTG TCA ATC AGA ACC TG-3'; R: 5'-ATG TAT TGT TCC TTG TAT AAG AAA TGG TGC-3'); $\beta$-actin (F: 5'-TTC AGG TTT ACT CAC GTC ATC C-3'; R: 5'CCA AAT GCG GCA TCT TCA AAC C-3'). Primers were synthesized by Beijing Sanbo Company (Beijing, China).

Human embryonic lung fibroblasts with population doublings of 21, 29, 44, 51, and 59 were selected, and when the fusion rate reached $90 \%$, total RNA was extracted with TRIzol. The RNA obtained was reverse-transcribed using PromegaImProm-II IM Reverse Transcriptase (Madison, WI, USA) according to manufacturer instructions. Polymerase chain reaction (PCR) amplification of related factors was performed after cDNA was obtained. The PCR system was performed according to previous study.

For western bot analysis, human embryonic lung fibroblasts with different population doublings were selected and lysed to extract proteins, and protein concentrations were determined as described in previous study. Samples were separated by $8 \%$ sodium dodecylsulfate (SDS)-polyacrylamide gel electrophoresis. Following electrophoresis, samples were transferred to a membrane. The membrane was sealed for $2 \mathrm{~h}$. The first antibody was added, the membrane was washed, and then the second antibody was added. The expression of the target protein was detected by chemiluminescence as described previously.

\section{RESULTS}

\section{Expression changes of $T R F 1$, tankyrase1, and POT1 in cell replicative senescence detected by reverse transcription-PCR (RT-PCR)}

The results from RT-PCR analysis showed that there were no significant changes in the mRNA levels of TRF1 during the aging process of human embryo lung fibroblasts with different population doublings (Figure 1A). Analysis of human embryonic lung fibroblasts with different population doublings showed that no significant changes in tankyrase 1 mRNA levels were detected (Figure 1B). In contrast, we found that the mRNA expression of POT1 showed a decreasing trend with increasing cell senescence (Figure 1C).

\section{Western blot determination of the expression changes of TRF1, tankyrase 1, and POT1 proteins in cell replicative senescence}

Through examination of the protein levels in human embryonic lung fibroblasts with different population doublings using western blot analysis, we found that the protein expres- 
sion levels of TRF1 showed an increasing trend with cell senescence (Figure 2A); however, the protein levels of tankyrase 1 did not change (Figure 2B).

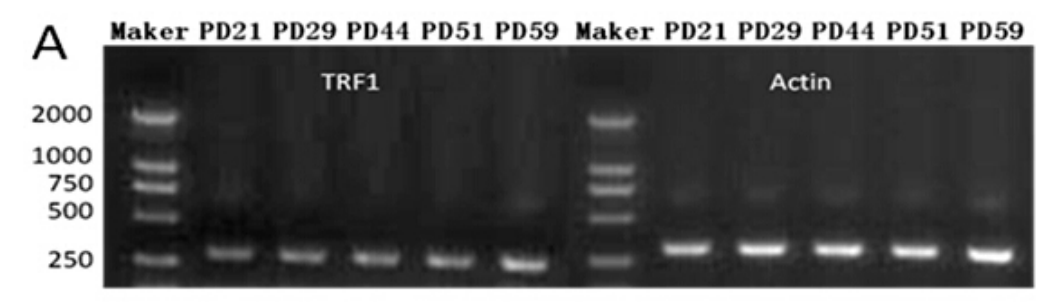

B

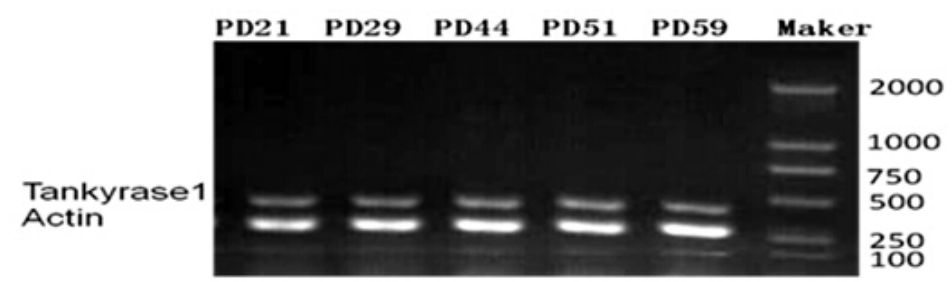

C

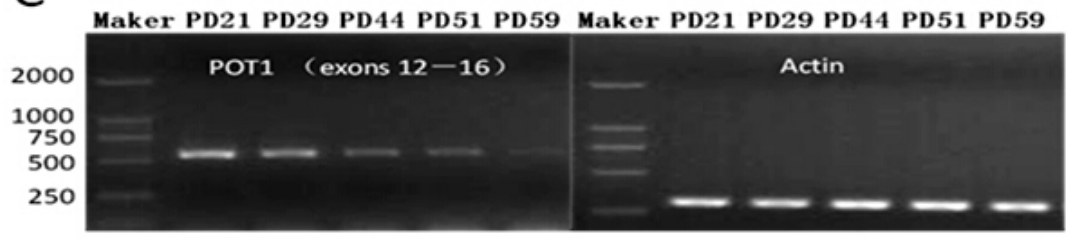

Figure 1. mRNA expression in different population doublings (PDs) of human embryo lung fibroblasts. The DNA marker used was DL-2000. PD21, PD29, PD44, PD51, and PD59 represent the 21, 29, 44, 51, and 59th PD of human embryonic lung fibroblast cells, respectively, as measured by polymerase chain reaction amplification. A. TRF1 mRNA expression changes. No difference was observed between different human embryo lung fibroblast PDs. B. Tankyrase $1 \mathrm{mRNA}$ expression changes. No difference was observed between different human embryo lung fibroblast PDs. C. POT1mRNA expression changes. A gradual decline with aging can be seen.

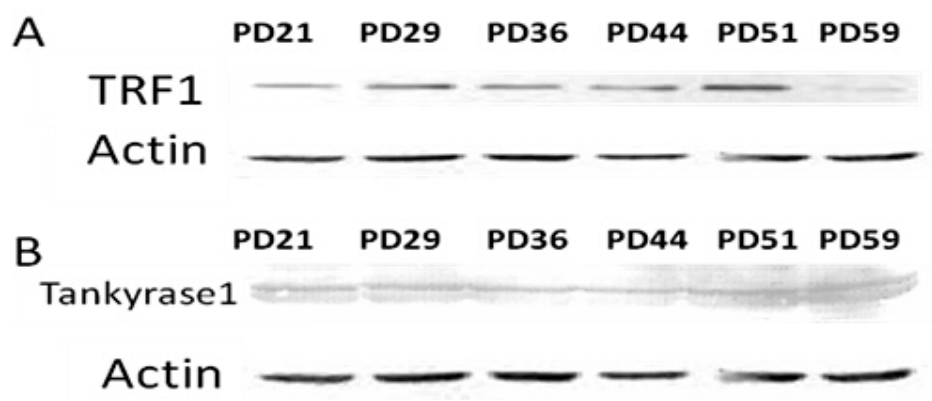

Figure 2. Protein expression in different population doublings (PDs) of human embryo lung fibroblasts. PD21, PD29, PD44, PD51, and PD59 represent the 21, 29, 44, 51, and 59th PD of human embryonic lung fibroblast cells, respectively, as detected by western blot. A. TRF1 protein expression changes. The expression levels associated with the aging of cells show a trend of gradual increase followed by a sharp decline in the amount of protein at PD59. B. Tankyrase 1 protein expression changes. No difference was observed between different human embryo lung fibroblast PDs. 


\section{Expression of telomerase RNA during cell senescence}

Through detection of the levels of telomerase RNA in the aging process of human embryo lung fibroblasts with different population doublings, we found that the mRNA expression of telomerase RNA showed an increasing trend with the cell senescence (Figure 3), indicating that telomerase RNA might also be involved in cellular replicative senescence.
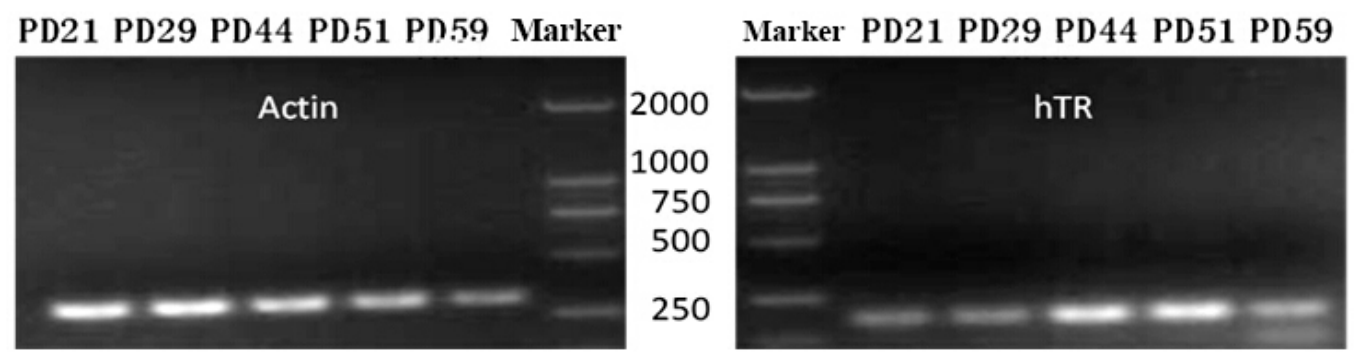

Figure 3. hTR expression changes. Left figure demonstrates $\beta$-actin mRNA expression; the right picture shows hTR mRNA expression level changes, as measured by polymerase chain reaction amplification. The DNA marker was DL-2000. Population doubling (PD)21, PD29, PD44, PD51, and PD59 represent the 21, 29, 44, 51, and 59th $\mathrm{PD}$ of human embryonic lung fibroblast cells, respectively. hTR expression first increased and then decreased with the aging of cells.

\section{Expression of p53 protein in cell senescence}

We examined the levels of p53 protein in the aging process of human embryo lung fibroblasts with different population doublings and found that the protein levels of $\mathrm{p} 53$ did not change significantly with cell senescence (Figure 4); however, whether the activity of p53 is increased remains to be determined.

\section{$\begin{array}{llllll}\text { PD21 } & \text { PD29 } & \text { PD36 } & \text { PD44 } & \text { PD51 } & \text { PD59 }\end{array}$}

\section{p53}

\section{Actin}

Figure 4. p53 protein expression changes. p53 and actin protein levels were detected by western blot. Population doubling (PD)21, PD29, PD44, PD51, and PD59 represent the 21, 29, 44, 51, and 59th PD of human embryonic lung fibroblast cells, respectively. No difference could be seen between different human embryo lung fibroblast PDs.

\section{DISCUSSION}

Telomere length is a "molecular clock" that controls the senescence of a cell. Telomere-associated protein has a role in the regulation of telomere length (Harly et al., 1990; Martínez and Blasco, 1997; Smogorzewska and Lange, 2004). Results have shown that human telomere binding protein 1 might play a role in this process in a variety of ways. 
The "protein counting model (mechanism) of telomere length regulation" hypothesis held the view that when telomere DNA combined with human telomere binding protein 1 reached a critical value, the signal that inhibited the activity of the telomerase compound would be produced and telomere elongation would be terminated. Consequently, if the chromosomes were not completely copied, they would be degraded by exonuclease or recombined, leading to shortened telomeres. In turn, the proportion of telomere combined with human telomere binding protein 1 would be also reduced; when this reached a critical value, the telomerase compound would be re-activated, and telomere length would be extended to a specific limit again. The cycle would then be repeated to maintain the stability of telomere length in the cancer cell. However, research on human telomere binding protein 1 regarding replicative senescence in normal cells has not been reported.

The results from this study demonstrated that no significant change could be detected in the mRNA expression levels of human telomere binding protein 1 in the aging process of human embryonic lung fibroblasts across different aged generations. This indicated that the transcription level of telomere binding protein 1 was not changed in the replicative senescence process of the cell, or, in turn, that normal cell senescence did not affect the expression of the human telomere binding protein 1 gene. Tankyrase 1 regulates the combination of human telomere binding protein 1 with telomeres. The results of our analyses showed that in the aging process, no change in the mRNA expression levels of tankyrase 1 in human embryonic lung fibroblasts across different aged generations could be detected, indicating that the transcription level of tankyrase 1 was not changed during the replicative senescence process of the cell, or, in turn, that normal cell senescence did not affect the expression of the tankyrase 1 gene. POT1 has been identified as an important protein for the maintenance of telomere overhangs. POT1 mRNA expression occurs in all tissues. Without POT1, the chromosome ends would become fused, leading to cell death. Thus, POT1 is considered a housekeeping gene that ensures the integrity of the chromosome ends. POT1 has five isoforms, four of which have been shown to be ubiquitously expressed, while splice variant 5 was only expressed in peripheral blood leukocytes. POT1 splice variant 2 missed exons 12a, while splice variant 5 missed exons 15a. If splice forms 2 and 5 exist, we would expect three bands to be amplified by RT-PCR according to the differing exon 12-16 regions. The three bands, from top to bottom, should be POT1, splice variants 5 and 2. By analyzing the mRNA levels of POT1 and its isoforms during the aging process of human embryo lung fibroblasts with different population doublings, we found that the mRNA expression of POT1 showed a decreasing trend with increasing cell senescence, and human embryonic lung fibroblast cells did not contain isoforms 5 and 2 . The mRNA expression level changes indicated that POT1 had a negative correlation with aging, and that cell senescence can impede the expression of POT1, potentially exacerbating telomere instability through the exposure of the telomere 3 ' overhang.

Our analyses found that cell senescence was associated with low expression of POT1, which might be an indicator of cell replicative senescence. The gene levels of human telomere binding protein 1 and tankyrase 1 did not change; hence, we further examined their protein expression. By analysis of human embryonic lung fibroblasts with different population doublings in the aging process, we found that the protein expression levels of TRF1 showed an increasing trend with cell senescence, indicating that expression levels of TRF1 had a positive correlation with cell senescence. Figure 2A showed that the expression of human telomere binding protein 1 in PD59 cells was low. We speculated that this occurred because the cells had begun to enter the cell death phase at this point. In contrast, it would be in the interest of 
younger cells to inhibit the aging process through high expression of human telomere binding protein 1 . However, after the telomeres had been shortened and were close to the "Hayflick limit", the apoptotic mechanism of the cell was initiated, and telomere binding protein 1was no longer highly expressed. Our analyses also determined that the protein levels of tankyrase 1 did not change across population doublings, indicating that there were no changes in the protein levels of tankyrase 1 in cell replicative senescence. That tankyrase 1, which regulates the binding of human telomere binding protein 1 to telomeric DNA, did not change in the cell replicative senescence process suggests that tankyrase 1 is not involved in the normal aging process and might just play a role in cell replication and telomere extension.

Telomerase RNA was first identified in 1995, and has been shown to exist in a variety of cells at constant levels. In normal cells, telomerase is inactive but its mRNA is expressed, raising the question of whether the telomerase RNA can affect the replicative senescence of cells. By determining the levels of telomerase RNA in the aging process of human embryo lung fibroblasts with different population doublings, we found that telomerase mRNA expression showed an increasing trend along with cell senescence, indicating that telomerase RNA might also be involved in the cell replicative senescence process. This result suggested that cell senescence causes survival pressure on the cells, resulting in the activation and promotion of telomerase RNA expression in order to alleviate the stress caused by the loss of telomeres.

Many recent studies have examined p53-induced cell aging, and researchers are actively exploring the genetic mechanisms of p53-induced cellular senescence. Studies have shown that p53 can accelerate aging; we therefore examined the levels of p53 protein during the aging process of human embryo lung fibroblasts with different population doublings and found that the protein levels of p53 did not change with cell senescence (Figure 4); however, whether its activity was increased remained to be determined.

We studied the relationship between cell replicative senescence and gene and protein expression levels of three telomere proteins closely related with telomere stability (telomere binding protein 1, tankyrase 1, and POT1). The results showed that cell senescence leads to the reduction of POT1 expression, which might be due to the 3 ' overhang shortening of telomeres upon continuous cell senescence and the concomitant reduced binding sites for POT1, leading to the reduction of POT1 transcription, thus exacerbating the instability of telomeres and accelerating the cell aging process. There were no changes in the gene expression of human telomere binding protein 1 or tankyrase 1, whereas the protein expression of human telomere binding protein 1 increased, suggesting that the cells might promote the translation of human telomere binding protein 1 in order to improve the stability of telomere DNA. Telomerase RNA was also found to be highly expressed during the aging process, which may be similar to the high expression of human telomere binding protein 1 , which might reflect the response of the cell to prevent the stress generated by aging. Although some studies have found that $\mathrm{p} 53$ can accelerate aging, our study did not suggest this; this phenomenon might be related to the phosphorylation of p53 and still needs further research.

In summary, we found that replicative senescence was closely associated with telomere related factors, of which human telomere binding protein 1, POT1, and tankyrase 1 might serve as housekeeping genes in the aging process, and their changes during this process might be the root causes of cell senescence. In addition, a large number of telomerase RNA was found to be expressed in normal cells; raising the question of the role of telomerase RNA in the aging process. Assessment of telomerase mRNA expression changes in cell senescence will be helpful to determine whether it participates in the aging process and plays an important 
role therein. Previous interactions of telomere-related factors as well as their relationships with telomeres were primarily studied in tumor cells; hence, expression changes of these related factors in normal cell senescence are not well understood. Therefore, we used the replicative senescence model of human embryonic lung fibroblasts as a basis, and analyzed TRF 1, POT1, tankyrase 1, and telomerase RNA at the molecular level to explore their expression changes during cell replicative senescence and to reveal their relationships with cellular replicative senescence.

\section{Conflicts of interest}

The authors declare no conflict of interest.

\section{ACKNOWLEDGMENTS}

The authors express their sincere thanks to all the teachers in the Key Laboratory Army of the Biochemical Department of Radiation Medicine at the Beijing Institute of Military Medical Sciences, and especially to Professor ZhengXiaofei for his contributions to the experimental design and thesis modification and his valuable advice. Sincere thanks are offered as well to all the teachers in the Clinical Research Center of the Inner Mongolia Medical University Hospital for their help and support.

\section{REFERENCES}

Abreu E, Aritonovska E, Reichenbach P, Cristofari G, et al. (2010). TIN2-tethered TPP1 recruits human telomerase to telomeres in vivo. Mol. Cell. Biol. 30: 2971-2982.

Blasco MA (2003). Mammalian telomeres and telomerase: why they matter for cancer and aging. Eur. J. Cell Biol. 82: 441-446.

Choi KH, Farrell AS, Lakamp AS and Ouellette MM (2011). Characterization of the DNA binding specificity of Shelterin complexes. Nucl. Acids Res. 39: 9206-9223.

Feng Z, Lin M and Wu R (2011). The regulation of aging and longevity: a new and complex role of p53. Genes Cancer 2: 443-452.

Giannone RJ, McDonald HW, Hurst GB, Shen R-F, et al. (2010). The protein network surrounding the human telomere repeat binding factors TRF1, TRF2, and POT1. PLoS One 5: e12407.

Harly CB, Futcher AB and Greider CW (1990). Telomeres shorten during aging of human fibroblasts. Nature 345: 458460.

Itahana K, Dimri G and Campisi J (2011). Regulation of cellular senescence by p53. Eur. J. Biochem. 268: 2784-2791.

Lundblad V (2003). Taking the measure. Nature 423: 926-927.

Martínez P and Blasco MA (1997). Telomeric and extra-telomeric roles for telomerase and the telomere-binding proteins. Nat. Rev. Cancer 11: 161-176.

Martínez P and Blasco MA (2010). Role of shelterin in cancer and aging. Aging Cell 9: 653-666.

Miller AS, Balakrishnan L, Buncher NA, Opresko PL, et al. (2012). Telomere proteins POT1, TRF1 and TRF2 augment long-patch base excision repair in vitro. Cell Cycle 11: 998-1007.

Smogorzewska A and de Lange T (2004). Regulation of telomerase by telomeric proteins. Annu. Rev. Biochem. 73: 177208.

Takai KK, Hooper S, Blackwood S, Gandhi R, et al. (2010). In vivo stoichiometry of shelterin components. J. Biol. Chem. 285: 1457-1467.

Takai KK, Kibe T, Donigian JR, Frescas D, et al. (2011). Telomere protection by TPP1/POT1 requires tethering to TIN2. Mol. Cell 44: 647-659.

Xie Y, Yang D, He Q and Songyang Z (2011). Zebrafish as a model system to study the physiological function of telomeric protein TPP1. PLoS One 6: e16440. 